\title{
A novel sequential algorithm for clutter and direct signal cancellation in passive bistatic radars
}

\author{
Farzad Ansari ${ }^{1}$, Mohammad Reza Taban² ${ }^{*}$ and Saeed Gazor ${ }^{3}$
}

\begin{abstract}
Cancellation of clutter and multipath is an important problem in passive bistatic radars. Some important recent algorithms such as the ECA, the SCA and the ECA-B project the received signals onto a subspace orthogonal to both clutter and pre-detected target subspaces. In this paper, we generalize the SCA algorithm and propose a novel sequential algorithm for clutter and multipath cancellation in the passive radars. This proposed sequential cancellation batch (SCB) algorithm has lower complexity and requires less memory than the mentioned methods. The SCB algorithm can be employed for static and non-static clutter cancellation. The proposed algorithm is evaluated by computer simulation under practical FM radio signals. Simulation results reveal that the SCB provides an admissible performance with lower computational complexity.
\end{abstract}

Keywords: Passive radar, Bistatic multipath, Clutter cancellation

\section{Introduction}

Passive bistatic radars use the reflected signals from independent transmitters as illuminators of opportunity. Passive radars stay hidden and cannot be identified or localized as they do not transmit signals while they detect aerial targets. In this type of radar, the utilized signals can be analogue TV [1, 2], FM radio [3], satellite [4], DVB-T [5], DAB [6] and GSM [7] which may be present in the space and can be treated as the transmitted signal. In general, the selection of suitable illumination signals depends on some parameters such as the coverage area of these transmitters, their power and their carrier frequency and bandwidth. Commercial FM radio stations are one of the best available signal sources which yield good performance for this purpose along with low implementation costs [3]. In particular, the high transmit powers of FM broadcast stations often allow detection ranges of approximately $250 \mathrm{~km}$ [8]. Figure 1 illustrates a common scenario that often occurs in the passive radars, where the passive radar is equipped with a receive reference antenna and a surveillance antenna.

*Correspondence: mrtaban@cc.iut.ac.ir

${ }^{2}$ Department of Electrical and Computer Engineering, Isfahan University of Technology, Isfahan 84156-83111, Iran

Full list of author information is available at the end of the article
The reference antenna is adjusted to receive only the direct path of the signal from the transmitter, while the surveillance antenna receives signals from all directions which includes signals not only from the direct path from the FM station but also from the reflections produced by targets and clutters. Using the ambiguity function based on the matched filters $[3,9]$, the Range-Doppler targets and clutter are detectable.

Before computing the ambiguity function, there are some challenges that must be resolved. For example, the power of the direct path signal is significantly higher than the received power from targets, and the signal received from the target and clutter often go through multipath unknown channels. Various methods have been proposed to confront these problems. Some of them have considered the problem as a composite hypothesis test and have attempted to design sub-optimal detectors such as generalized likelihood ratio test for target detection in the presence of the interference $[10,11]$. Some others have employed adaptive filters to estimate the clutter and direct path signal components in order to cancel them $[12,13]$. However, an important class of methods is based on the projection of the received signal onto a subspace orthogonal to both the clutter and the pre-detected targets. The ECA, SCA and ECA-B are among these methods [14-16].
Springer Open (c) The Author(s). 2016 Open Access This article is distributed under the terms of the Creative Commons Attribution 4.0 International License (http://creativecommons.org/licenses/by/4.0/), which permits unrestricted use, distribution, and reproduction in any medium, provided you give appropriate credit to the original author(s) and the source, provide a link to the Creative Commons license, and indicate if changes were made. 


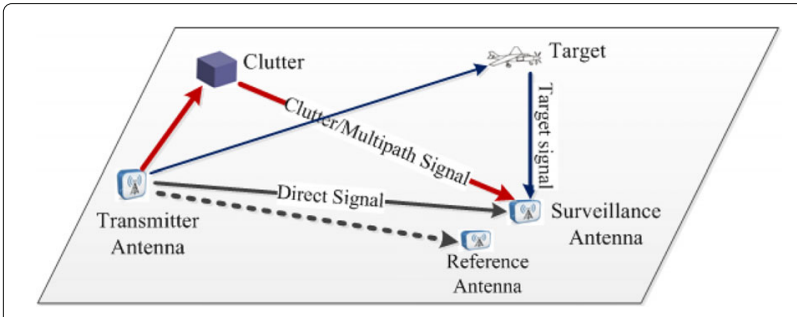

Fig. 1 A common scenario of passive radars

Recently, a version of ECA (ECA-S) has been proposed in [17].

In this paper, we propose a novel algorithm for clutter and multipath cancellation for the passive radars by generalization of some recent algorithms which we call as the sequential cancellation batch (SCB) algorithm. Our simulation results show that the proposed SCB outperforms or performs as good as the mentioned state-of-theart methods, depending on the conditions. Furthermore, the proposed SCB requires lesser memory than these existing state-of-the-art methods. Our simulations show that after clutter and direct signal cancellation using the SCB algorithm, weak targets likely are not detectable. Hence, in this paper, we use CLEAN algorithm [18-20] for weak target detection. Although in this paper, we concentrate on the use of commercial FM radio signals, it should be noted that the proposed method (SCB) can be applied to any transmission of opportunity, such as GSM transmissions, DAB or DVB-T and satellites. Indeed, the choice of FM transmissions arguably results in waveforms with the worst ambiguity properties for target detection.

The paper is organized as follows. Section 2 presents the signal model and ambiguity function. Section 3 introduces the ECA and SCA algorithms and describes the proposed SCB technique, and in Section 4, three tests are introduced for comparison of the performance of algorithms. Finally, Section 5 is our conclusions.

Notations: Throughout this paper, we use boldface lower case and capital letters to denote vector and matrix, respectively. We use $\mathcal{O}($.$) as the complexity order of algo-$ rithms. diag $(., \ldots,$.$) denotes diagonal matrix containing$ the elements on the main diameter. $\mathbf{0}_{N \times R}$ is an $N \times R$ zero matrix and $\mathbf{I}_{N}$ is an $N \times N$ identical matrix. Also (.) ${ }^{T},(.)^{*}$ and $(.)^{H}$ stand for the transpose, conjugate and Hermitian of a matrix or vector, respectively. The operator $\lfloor$. denotes the integer part (or floor) of a number.

\section{Signal model and ambiguity function}

The FM radio signals used in passive radar are in the 88 - to $108-\mathrm{MHz}$ band. For example, in Fig. 2, the spectrum of a commercial FM signal is showed that is used for simulation scenario.

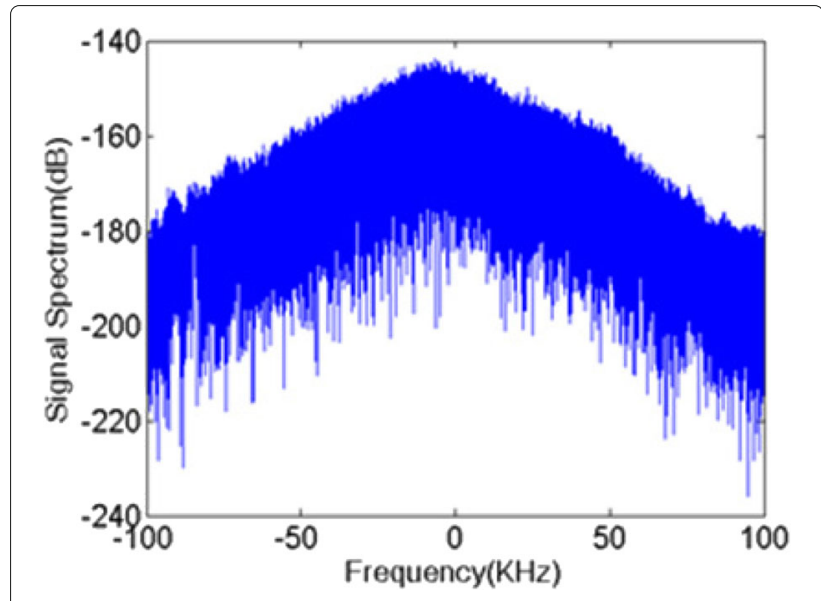

Fig. 2 Spectrum of FM signal used for simulated scenario

As seen in Fig. 1, two required signals are used for interference cancellation algorithms. One is the main received signal from the surveillance antenna and another is an auxiliary signal yielded from the reference antenna. If $T_{\text {int }}$ is the duration time of signal observation, the received signal $s_{\text {sur }}(t)$ at the surveillance antenna is modelled as:

$$
\begin{aligned}
s_{\mathrm{sur}}(t)= & A_{\mathrm{sur}} d(t)+\sum_{m=1}^{N_{T}} a_{m} d\left(t-\tau_{m}\right) e^{j 2 \pi f_{\mathrm{d} m} t} \\
& +\sum_{i=1}^{N_{C}} c_{i}(t) d\left(t-\tau_{c i}\right)+n_{\mathrm{sur}}(t), 0 \leq t \leq T_{\mathrm{int}},
\end{aligned}
$$

where $d(t)$ is the direct transmitted signal that is multi-

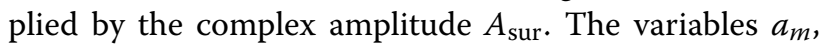
$\tau_{m}$ and $f_{\mathrm{d} m}$ are the complex amplitude, delay and Doppler frequency of the $m$ th target signal $\left(m=1, \ldots, N_{T}\right)$, respectively, that is $N_{T}$ is the number of targets. $c_{i}(t)$ and $\tau_{c i}$ are the complex amplitude function and delay of the $i$ th clutter $\left(i=1, \ldots, N_{C}\right)$, that is $N_{C}$ is the number of clutters. All delays are calculated with respect to the direct signal. $n_{\text {sur }}(t)$ is the thermal noise contribution at the receiver antenna.

The complex amplitudes $c_{i}(t)$ are considered slowly varying functions of time, so that they can be represented by only a few frequency components around zero Doppler:

$$
c_{i}(t)=c_{i} e^{j 2 \pi f_{c i} t},
$$

where $f_{c i}$ and $c_{i}$ are Doppler shift and complex amplitude of the $i$ th clutter $\left(i=1, \ldots, N_{C}\right)$, respectively. In the same way, the received signal $s_{\mathrm{ref}}(t)$ at the reference antenna is:

$$
s_{\mathrm{ref}}(t)=A_{\mathrm{ref}} d(t)+n_{\mathrm{ref}}(t),
$$

where $A_{\text {ref }}$ is a complex amplitude and $n_{\text {ref }}(t)$ is the thermal noise contribution at the reference antenna. 
The samples collected at the surveillance channel at the time instants $t_{n}=n T_{s},(n=0,1, \ldots, N-1)$ are arranged in a $N \times 1$ vector $\mathbf{s}_{\text {sur }}$, where $T_{s}$ is the sampling time and $N$ is the number of samples to be integrated. The sampling time is selected greater than the resolution time (i.e. $T_{s}>1 / B$ where $B$ is the system bandwidth). Similarly, we collect $N+R$ samples of the signal at the reference channel in a $(N+R) \times 1$ vector $\mathbf{s}_{\text {ref. }}$. We use the ambiguity function for evaluation of the interference cancellation algorithms and target detection. The discrete ambiguity function equation is as follows [9]:

$$
\xi[l, p]=\sum_{i=0}^{N-1} s_{\mathrm{sur}}[i] s_{\mathrm{ref}}^{*}[i-l] e^{-\frac{j 2 \pi p i}{N}} \quad, \quad \begin{gathered}
l=0, \ldots, R, \\
p=0, \ldots, P-1
\end{gathered}
$$

where $s_{\text {sur }}[i]$ and $s_{\text {ref }}[i]$ denote $s_{\text {sur }}\left(t_{i}\right)$ and $s_{\text {ref }}\left(t_{i}\right)$, respectively. Consider that the discrete delay $l$ corresponds to the delay $T[l]=l T_{s}$ and $R$ is maximum delay bin of clutter. Similarly, the discrete Doppler frequency bin $p$, corresponds to the Doppler frequency $f_{d}[p]=p /\left(N T_{s}\right)$ and $P$ is maximum Doppler bin of clutter.

\section{Clutter and direct signal cancellation}

In this section, first we introduce two known algorithms ECA and SCA for clutter and direct signal cancellation in passive bistatic radars. Then the proposed algorithm is presented.

\subsection{Extensive cancellation algorithm (ECA)}

The ECA is an effective way for clutter and direct signal cancellation in the passive radars and is based on the leastsquares (LS) estimation [14]. If the surveillance vector $\mathbf{s}_{\text {sur }}$ is modelled with respect to the reference vector $\mathbf{s}_{\text {ref }}$ linearly, the objective function in the LS estimation can be represented as follows:

$$
\min _{\boldsymbol{\theta}}\left\|\mathbf{s}_{\mathrm{sur}}-\mathbf{H} \boldsymbol{\theta}\right\|
$$

where $\theta$ is the model parameters vector corresponding to the likely clutters and $\mathbf{H}$ is a known matrix depending on the positive integer $p$ as:

$$
\mathbf{H}=\mathbf{B}\left[\begin{array}{llll}
\Delta_{-p} \mathbf{s}_{\mathrm{ref}} & \ldots \boldsymbol{\Delta}_{-1} \mathbf{s}_{\mathrm{ref}} & \mathbf{s}_{\mathrm{ref}} & \left.\boldsymbol{\Delta}_{1} \mathbf{s}_{\mathrm{ref}} \ldots \boldsymbol{\Delta}_{p} \mathbf{s}_{\mathrm{ref}}\right]
\end{array}\right.
$$

Here, $\mathbf{B}$ is an incidence matrix that selects only the last $N$ rows of the next multiplied matrix and has the below form:

$$
\mathbf{B}=\left[\mathbf{0}_{N \times R} \vdots \mathbf{I}_{N}\right]
$$

$\boldsymbol{\Delta}_{p}$ is a diagonal matrix making the phase shift corresponding to the $p$ th Doppler bin, as:

$$
\boldsymbol{\Delta}_{p}=\operatorname{diag}\left(1, e^{j 2 \pi p T_{s}}, \ldots, e^{j 2 \pi(N+R-1) p T_{s}}\right) .
$$

Also, $\mathbf{s}_{\text {ref }}=\left[\begin{array}{lllll}\mathbf{s}_{\text {ref }} & \mathbf{D s}_{\text {ref }} & \mathbf{D}^{2} \mathbf{s}_{\text {ref }} \ldots \mathbf{D}^{k-1} \mathbf{s}_{\text {ref }}\end{array}\right]$, where $\mathbf{D}$ is a $0 / 1$ permutation matrix that imposes a delay unit to the next multiplied vector and $k$ indicates the maximum amount of delay in clutter samples. Indeed, the columns of $\mathbf{s}_{\text {ref }}$ are the delayed versions of the zero-Doppler reference signal. The columns of matrix $\mathbf{H}$ present a basis for the $M$-dimensional clutter subspace, where $M=(2 p+1) k$. The solution of (7) yields $\hat{\boldsymbol{\theta}}=\left(\mathbf{H}^{\mathrm{H}} \mathbf{H}\right)^{-1} \mathbf{H}^{\mathrm{H}} \mathbf{s}_{\text {sur }}$; therefore, the received signal after cancellation of direct signal and clutter can be obtained as:

$$
\mathbf{s}_{E C A}=\mathbf{s}_{\text {sur }}-\mathbf{H} \hat{\boldsymbol{\theta}}=\left(\mathbf{I}_{N}-\mathbf{H}\left(\mathbf{H}^{\mathrm{H}} \mathbf{H}\right)^{-1} \mathbf{H}^{\mathrm{H}}\right) \mathbf{s}_{\text {sur }}=\mathbf{P}_{0} \mathbf{s}_{\text {sur }} .
$$

The computational complexity of the ECA algorithm is $\mathcal{O}\left(N M^{2}+M^{3}\right)$. This complexity is high because the estimation of vector $\boldsymbol{\theta}$ requires the inversion of the matrix $\mathbf{H}^{\mathrm{H}} \mathbf{H}$ with dimensions $M \times M$.

\subsection{Sequential cancellation algorithm (SCA)}

Aiming at reducing the computational load of the ECA algorithm described in Section 3.1, a sequential solution algorithm has been offered in [14] for clutter and direct signal cancellation, called SCA.

Consider the matrix $\mathbf{H}=\left[\begin{array}{llll}\mathbf{x}_{0} & \mathbf{x}_{1} & \cdots & \mathbf{x}_{M-1}\end{array}\right]$, where $\mathbf{x}_{i}$ is the $(i+1)$ th column of $\mathbf{H}$. The sequential equations of the SCA algorithm are as follows:

- Start with initial equations as:

$$
\begin{array}{r}
\mathbf{P}_{M}=\mathbf{I}_{N}, \\
\overline{\mathbf{s}}_{\text {sur }}^{(M)}=\mathbf{s}_{\text {sur }} .
\end{array}
$$

- Then, the output vector of SCA algorithm is obtained by implementing the below recursive equations for $i=M, \ldots, 2,1$ respectively:

$$
\begin{array}{r}
\overline{\mathbf{x}}_{j}^{(i)}=\mathbf{P}_{i} \mathbf{x}_{j} \quad \text { for } \quad j=0,1, \ldots, i-1, \\
\mathbf{Q}_{i}=\left[\begin{array}{r}
\left.\mathbf{I}_{N}-\frac{\overline{\mathbf{x}}_{i-1}^{(i)} \overline{\mathbf{x}}_{i-1}^{(i) \mathrm{H}}}{\overline{\mathbf{x}}_{i-1}^{(i)} \overline{\mathbf{x}}_{i-1}^{(i)}}\right], \\
\mathbf{P}_{i-1}=Q_{i} P_{i}
\end{array}\right.
\end{array}
$$

- In each step of the above equations, the received signal can be improved one level as:

$$
\mathbf{s}_{\text {sur }}^{(i-1)}=\mathbf{P}_{i-1} \mathbf{s}_{\text {sur }}=\mathbf{Q}_{i} \mathbf{s}_{\text {sur }}^{(i)} \text {. }
$$

- After finishing the above loop, by using the final projection matrix $\mathbf{P}_{0}$, the output vector $\mathbf{s}_{\mathrm{SCA}}$ is obtained as follows:

$$
\mathbf{s}_{\mathrm{SCA}}=\mathbf{s}_{\text {sur }}^{(0)}=\mathbf{P}_{0} \mathbf{s}_{\text {sur }} \text {. }
$$


A schematic plan of the SCA algorithm containing the clutter and direct signal cancellation is shown in Fig. 3.

Almost all steps of a SCA algorithm have been shown in this figure. It is possible to limit the computational of the cancellation algorithm by arresting it after stage $S(S<$ $M)$. The computational complexity of the SCA algorithm limited to $S$ stage is $\mathcal{O}(N M S)$, which can be significantly smaller than the computational cost of the corresponding complete ECA algorithm.

\subsection{Sequential cancellation batch (SCB) algorithm}

In order to improve the cancellation performance with a limited computational load, a modification of the SCA is proposed which is called SCB. The received signal at the surveillance antenna is divided into sections with length $T_{B}$. If the entire length of the surveillance antenna signal is $T_{\text {int }}$, the total number of samples of the signal at the antenna will be $N=\left\lfloor T_{\operatorname{int}} f_{s}\right\rfloor$, where $f_{s}$ is the sampling frequency. The signal is divided into $b$ packets with $N_{B}=\lfloor N / b\rfloor$ available samples. First, the SCA algorithm is applied to each of these packets distinctly. The output of the SCA algorithm on each packet is a vector removed of the clutter and direct signal. Then, the main cleaned vector is obtained from the union of these sub-vectors. Finally, the main vector can be used for computing and plotting the ambiguity diagram and target detection.

In this manner, vectors $\mathbf{s}_{\operatorname{surv}(j)}$ and $\mathbf{s}_{\mathrm{ref}(j)}$ corresponding to the $(j+1)$ th packet are defined as follows for $j=$ $0,1,2, \ldots, b-1$

$\mathbf{s}_{\text {surv }(j)}=\left[\mathbf{s}_{\text {sur }}\left[j N_{B}\right] \quad \mathbf{s}_{\text {sur }}\left[j N_{B}+1\right] \ldots \mathbf{s}_{\text {sur }}\left[(j+1) N_{B}-1\right]\right]^{\mathrm{T}}$,

$\mathbf{s}_{\mathrm{ref}(j)}=\left[\mathbf{s}_{\mathrm{ref}}\left[j N_{B}-R\right] \mathbf{s}_{\mathrm{ref}}\left[j N_{B}-R+1\right] \ldots \mathbf{s}_{\mathrm{sur}}\left[(j+1) N_{B}-1\right]\right]^{\mathrm{T}}$.

If the output of the SCA algorithm on the $j$ th packet denotes vector $\mathbf{s}_{\mathrm{SCA}(j)}$, the total output $\mathbf{s}_{\mathrm{SCB}}$ removed from the clutter and direct signal is obtained as:

$$
\mathbf{s}_{\mathrm{SCB}}=\left[\begin{array}{llll}
\mathbf{s}_{\mathrm{SCA}(0)}^{\mathrm{T}} & \mathbf{s}_{\mathrm{SCA}(1)}^{\mathrm{T}} & \cdots & \mathbf{s}_{\mathrm{SCA}(b-1)}^{\mathrm{T}}
\end{array}\right]^{\mathrm{T}} .
$$

A schematic plan of the SCB algorithm is shown in Fig. 4. If we replace the applied SCA method with ECA

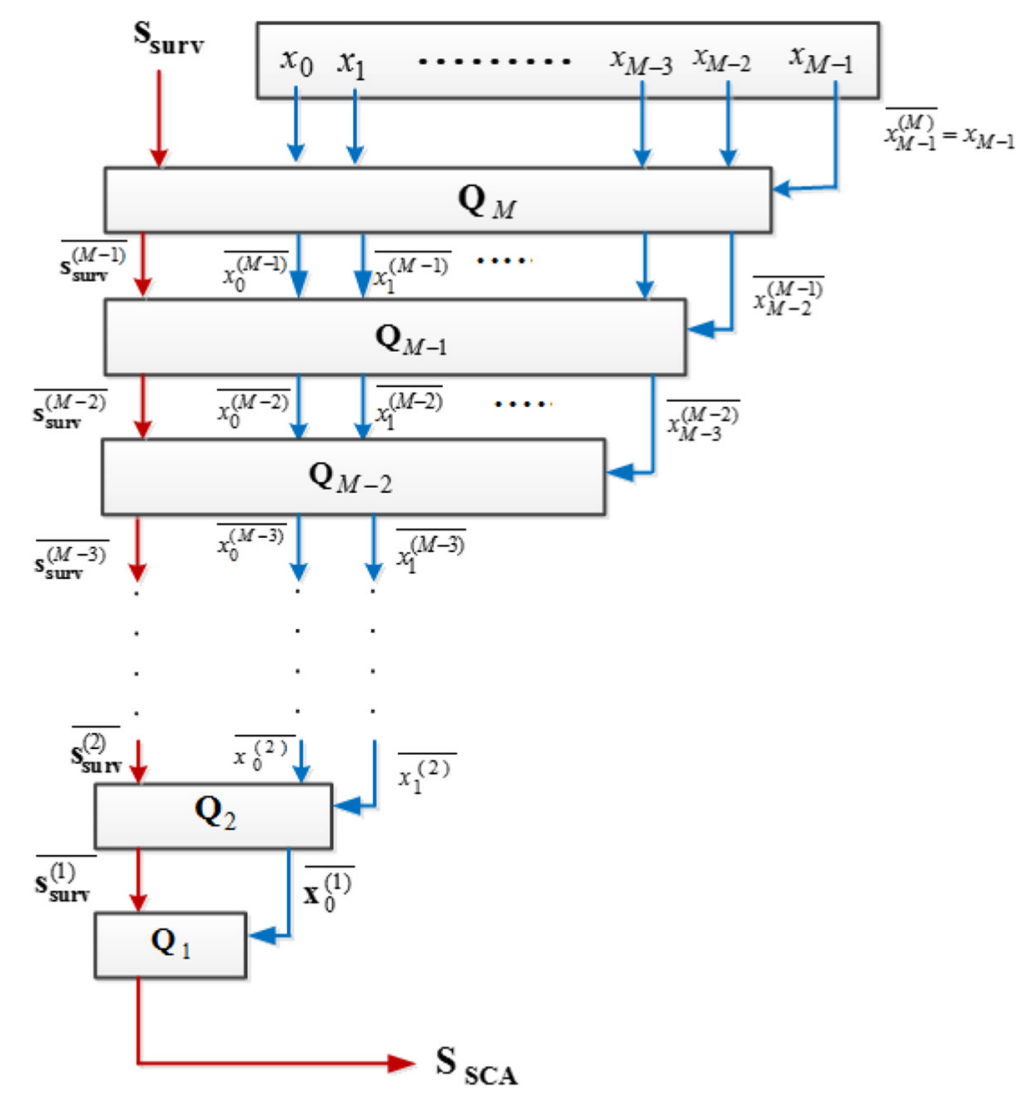

Fig. 3 Sketch of the sequential cancellation algorithm 


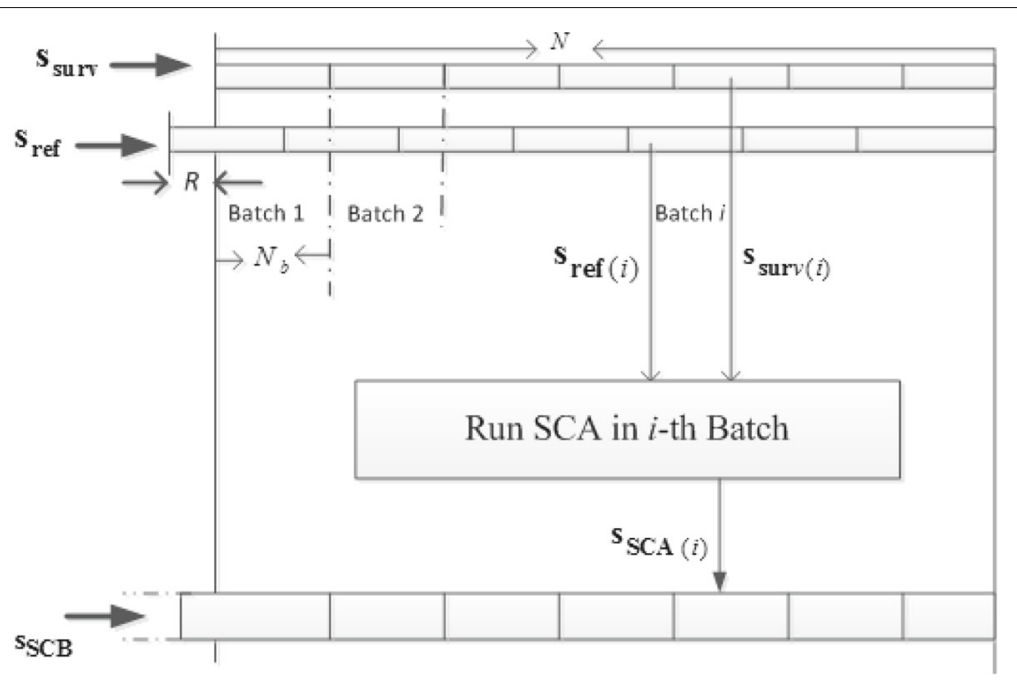

Fig. 4 A sketch of the SCB approach

method in the SCB algorithm (instead of the SCA block that runs on the $i$ th batch in the block diagram of Fig. 4), the ECA-B method will be achieved which is explained in [15] fully.

We use the observation or CLEAN algorithm [18-21] for detection of the weak target. The Doppler frequency $\left(\hat{f}_{i 1}\right)$, delay $\left(\hat{\tau}_{i 1}\right)$ and amplitude $\left(\hat{A}_{i 1}\right)$ of the $i$ th strong target $\left(i=1, \ldots, N_{s 1}\right)$ can be extracted based on the information of location of this strong target in the ambiguity function where $N_{s 1}$ is the number of strong targets which are detected after SCB algorithms. Then, the estimated echoes of the strong targets are subtracted from $s_{S C B}(t)$ as follows:

$$
s_{\text {sur }}^{1}(t)=s_{S C B}(t)-\sum_{i=1}^{N_{s 1}} \hat{A}_{i 1} d\left(t-\hat{\tau}_{i 1}\right) e^{2 \pi j \hat{f}_{i 1} t},
$$

where $s_{S C B}(t)$ is the signal removed from clutter and direct signal by the SCB algorithm. By computing the ambiguity function of $s_{\text {sur }}^{1}(t)$, the weak targets can appear. In the next processing, the estimated echoes of new targets (the weak targets in ambiguity function of $\left.s_{\text {sur }}^{1}(t)\right)$ are subtracted from $s_{\text {sur }}^{1}(t)$ as follows:

$$
s_{\text {sur }}^{2}(t)=s_{\text {sur }}^{1}(t)-\sum_{i=1}^{N_{s 2}} \hat{A}_{i 2} d\left(t-\hat{\tau}_{i 2}\right) e^{2 \pi j \hat{f}_{i 2} t} .
$$

Here, the Doppler frequency $\left(\hat{f}_{i 2}\right)$, delay $\left(\hat{\tau}_{i 2}\right)$ and amplitude $\left(\hat{A}_{i 2}\right)$ of each weak target can be extracted based on the information of location of these weak targets in the ambiguity function of $s_{\text {sur }}^{1}(t)$, and $N_{s 2}$ is the number of weak targets which are detected in ambiguity function of $s_{\text {sur }}^{1}(t)$. The observation algorithm is repeated as follows:

$$
s_{\mathrm{sur}}^{j}(t)=s_{\mathrm{sur}}^{j-1}(t)-\sum_{i=1}^{N_{s j}} \hat{A}_{i j} d\left(t-\hat{\tau}_{i j}\right) e^{2 \pi \hat{f_{i j}} t}, j=2,3, \ldots
$$

where $\hat{f}_{i j}, \hat{\tau}_{i j}$ and $\hat{A}_{i j}$ are the the Doppler frequency, delay and amplitude of unregarded weak targets which appeared in the $(j-1)$ th stage and detected using the ambiguity function of $s_{\text {sur }}^{j-1}(t)$. The algorithm is ended when the below inequality occurs:

$$
\frac{\max \left(\xi\left(\tau_{d}, f_{d}\right)\right)-\min \left(\xi\left(\tau_{d}, f_{d}\right)\right)}{\max \left(\xi\left(\tau_{d}, f_{d}\right)\right)}<\eta,
$$

where $\xi\left(\tau_{d}, f_{d}\right)$ is the ambiguity function of $s_{\text {sur }}^{j}(t)$ at position $\left(\tau_{d}, f_{d}\right)$ and $\eta$ is a small value selected between zero and one in our simulations.

The computational complexity of the SCB algorithm in each batch is $\mathcal{O}\left(N_{B} M S\right)$. This means that the SCB algorithm requires lesser memory than the ECA and SCA algorithms. When the SCB algorithm is run on $b$ batches, the computational complexity will be $\mathcal{O}\left(b N_{B} M S\right)$ which is equal to the SCA algorithm because $b N_{B}$ equals $N$.

We remind that the computational complexity of ECAB method is $\mathcal{O}\left(N M^{2}+M^{3}\right)$ similar to the ECA method; but its required memory is $\mathcal{O}\left(N_{B} M^{2}+M^{3}\right)$ which is less than that of ECA. Anyway, both computational complexity and required memory of the proposed SCB method are considerably less than those of ECA-B method. 


\section{Results and discussion}

In this section, we investigate the performance of the proposed algorithm and compare it with the ECA, SCA and ECA-B methods. For this purpose, we use two different Doppler-delay scenarios. First, in scenario \#1 represented in Fig. 5, we consider nine clutters in the form of blue stars and three targets in the form of red circles.

The clutter and target specifications are shown in Tables 1 and 2, respectively. Also, the signal-to-noise ratio (SNR) of the direct signal is assumed to be $60 \mathrm{~dB}$.

Figure 6 shows the ambiguity function of the received signal in scenario \#1, in a two-dimensional (2-D) mode without removing the direct signal and clutter. In Fig. 6a, it is seen that the peaks of targets are masked by the peaks of direct signal and clutters, and the targets are not distinguishable. In Fig. 6b, the output of ambiguity function is drawn for $l=0$. In this figure, the strong peak corresponding to the direct signal (with zero Doppler and zero delay) is presented obviously. The output of ambiguity function for $p=0$ is also presented in Fig. 6c. In this figure, it is shown that the clutter is delayed up to $0.25 \mathrm{~ms}$, and most of the amounts of ambiguity function are in zero delay, indicating the direct path signal.

First, we implement the ECA algorithm under scenario \#1. Figure 7 a shows the 2-D ambiguity function of the received signal after the direct signal and all echoes of clutter cancellation by the ECA algorithm. The simulation conditions are $k=50$ and Doppler bin $(-1,0,1)$, where $p$ is 1 . As seen in Fig. 7a, the two strong targets now appear, but the weak target is still not detectable. Figure $7 \mathrm{~b}$ shows the ambiguity function versus delay in Doppler shift $l=0$.

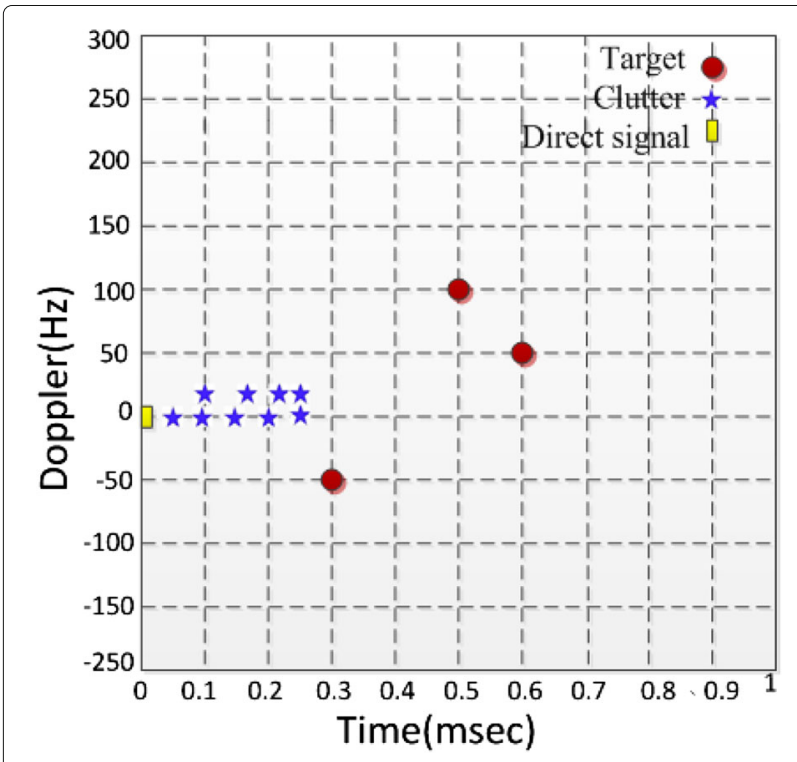

Fig. 5 Representation of scenario \#1
Table 1 Clutter echo parameters in scenario \#1

\begin{tabular}{llllllllll}
\hline Clutter & $\# 1$ & $\# 2$ & $\# 3$ & $\# 4$ & $\# 5$ & $\# 6$ & $\# 7$ & $\# 8$ & $\# 9$ \\
\hline Delay (ms) & 0.05 & 0.1 & 0.15 & 0.2 & 0.25 & 0.1 & 0.17 & 0.22 & 0.25 \\
Doppler (Hz) & 0 & 0 & 0 & 0 & 0 & 1 & 1 & 1 & 1 \\
CNR (dB) & 40 & 30 & 20 & 10 & 5 & 27 & 18 & 8 & 5 \\
\hline
\end{tabular}

This figure shows that the direct signal and all clutters corresponding to the delays inside the first $k$ bins have been removed.

Then, the SCB algorithm is simulated based on scenario \#1. The information required for the simulation is shown in Table 3. Figure 8a shows the 2-D ambiguity function of the received signal after cancelling all the clutters and direct signal using the SCB algorithm. This simulation has been prepared with $S=100$, Doppler bin $(-1,0,1)$ and $k=50$. The cancellation of the direct signal and clutter causes the strong targets to be seen better, and by using a simple detector such as the cell-averaging constant false-alarm rate (CA-CFAR), they can simply be detected. Nonetheless, the weak target has not been detected. Figure $8 \mathrm{~b}, \mathrm{c}$ shows the ambiguity function versus delay for Doppler shifts -50 and $100 \mathrm{~Hz}$, and Fig. 8d, e shows the ambiguity function versus Doppler for delays 0.3 and $0.5 \mathrm{~ms}$, respectively. In these four figures, it is seen that unlike the weak targets, the locations of two strong targets are shown obviously.

For illustrating the modified SCB method equipped with CLEAN technique, Fig. 9a shows the 2-D ambiguity function output of received signal after removing the direct signal and all the clutters by the SCB algorithm, and the strongest target using the observation algorithm (consider that Fig. 9 presents the ambiguity function of $\left.s_{\text {sur }}^{1}(t)\right)$. Here, the weak target now appears as a strong peak. Figure $9 \mathrm{~b}$ shows the ambiguity function versus delay for Doppler $50 \mathrm{~Hz}$ and Fig. 9c shows the ambiguity function versus Doppler for delay $0.6 \mathrm{~ms}$. In these figures, the location of the weak target is observed obviously.

In the following, three tests are introduced for evaluation of SCB in comparison with ECA, SCA and ECA-B algorithms.

\subsection{Evaluation using $C A$ and TA tests}

In this section, the CA and TA tests are introduced for comparing the clutter and direct signal cancellation

Table 2 Target echo parameters in scenario \#1

\begin{tabular}{llll}
\hline Target & $\# 1$ & $\# 2$ & $\# 3$ \\
\hline Delay $(\mathrm{ms})$ & 0.3 & 0.5 & 0.6 \\
Doppler $(\mathrm{Hz})$ & -50 & 100 & 50 \\
SNR $(\mathrm{dB})$ & 4 & 2 & -10 \\
\hline
\end{tabular}




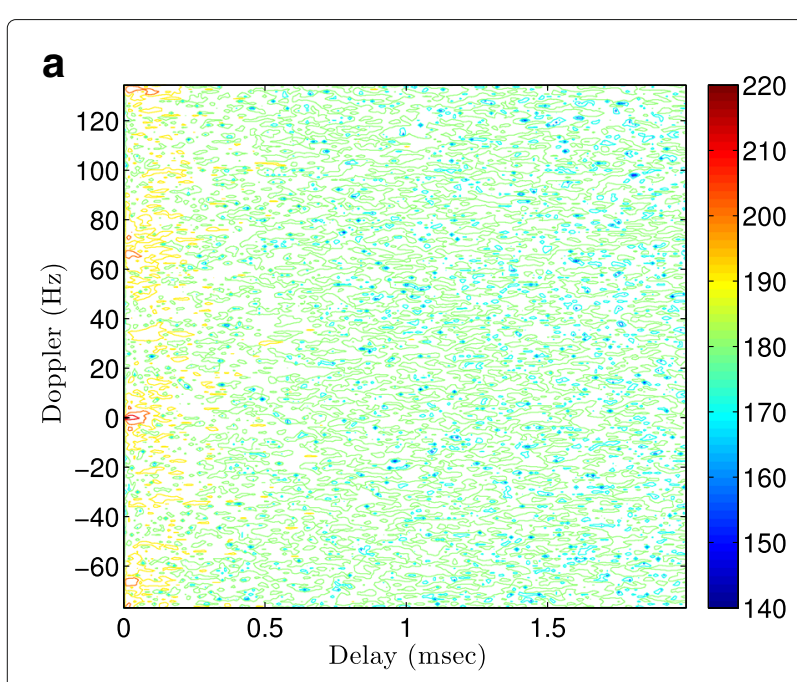

b

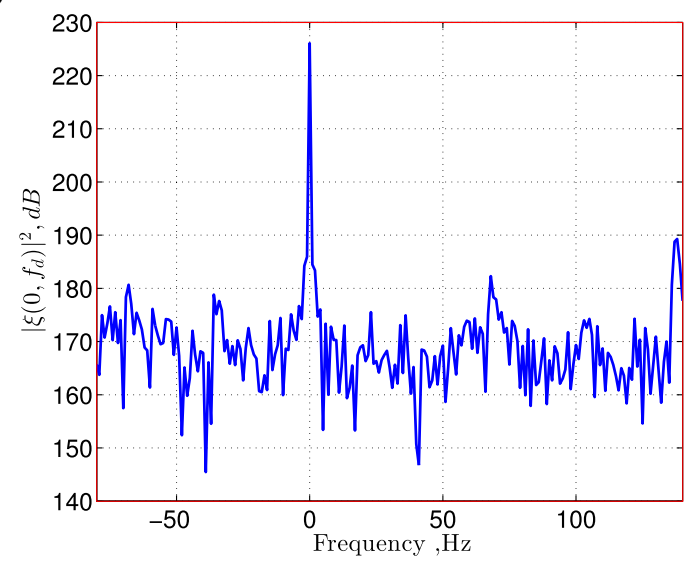

C

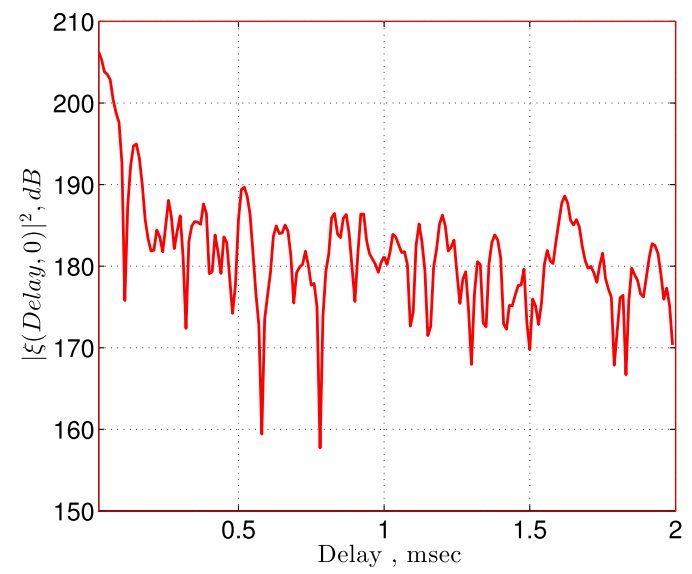

Fig. 6 The ambiguity function output in $\mathrm{dB}$ before cancellation in scenario \#1. a 2-D output. b Section at delay 0. c Section at Doppler 0

algorithms. Initially, the CA and TA are written as follows:

$$
\mathrm{CA}=10 \log \left(\frac{\text { input clutter amplitude peak }}{\text { output clutter amplitude peak }}\right)
$$

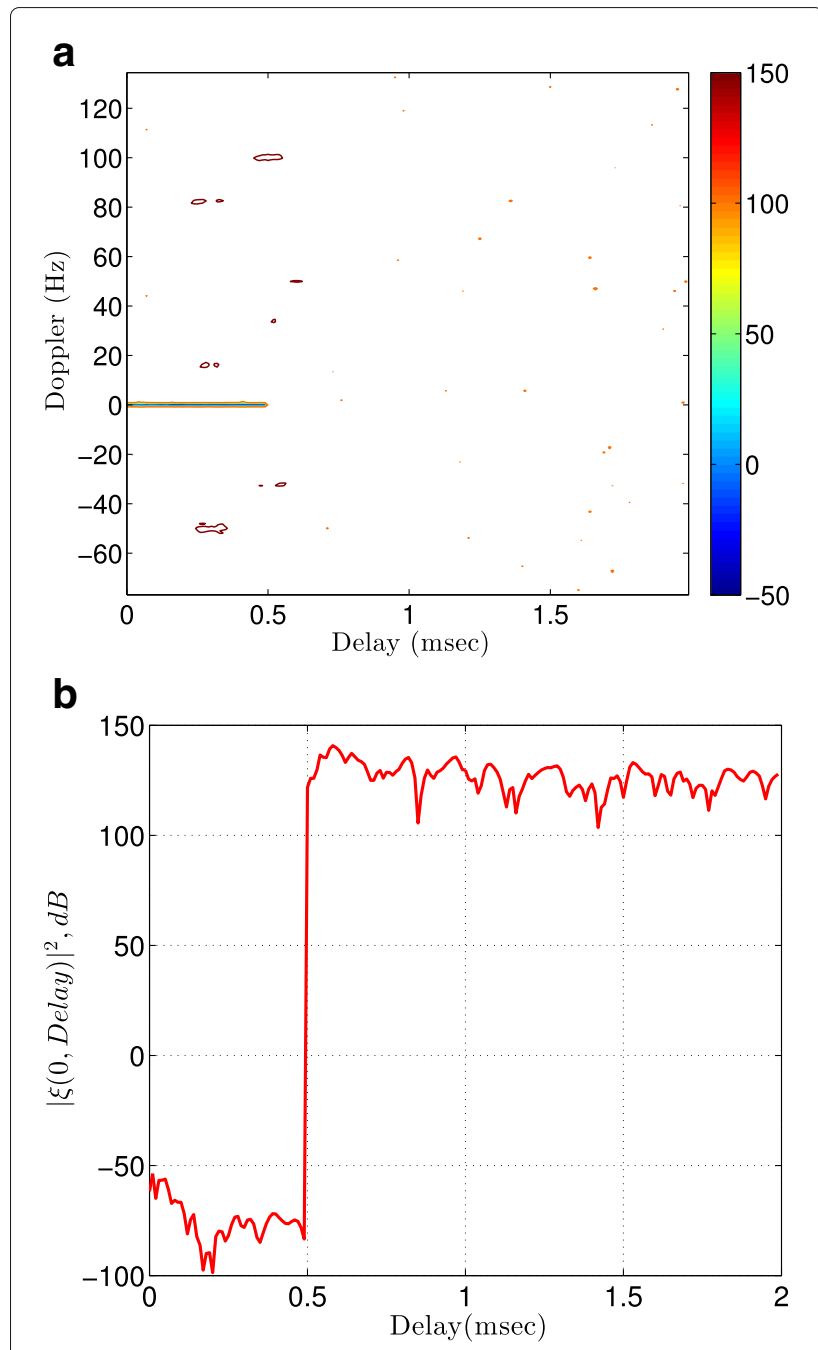

Fig. 7 The ambiguity function output in $\mathrm{dB}$ after direct signal and all clutter cancellation ( $k=50, p=1$ and $M=150$ ) by the ECA algorithm in scenario \#1. a 2-D output. b Section at $I=0$

$$
\mathrm{TA}=10 \log \left(\frac{\text { input target amplitude peak }}{\text { output target amplitude peak }}\right),
$$

where, the phrases "input clutter/target amplitude peak" and "output clutter/target amplitude peak" indicate the amplitude of clutter/target before and after the clutter and direct signal cancellation, respectively. For evaluating the SCB algorithm in comparison with the ECA, SCA and ECA-B algorithms using the CA and

Table 3 Selected parameters for simulation of the SCB algorithm

\begin{tabular}{lll}
\hline Observation time & $T_{\text {int }}$ & $1 \mathrm{~s}$ \\
\hline Sampling time & $T_{\mathrm{s}}$ & $0.005 \mathrm{~ms}$ \\
Number of batch & $b$ & 10 \\
\hline
\end{tabular}



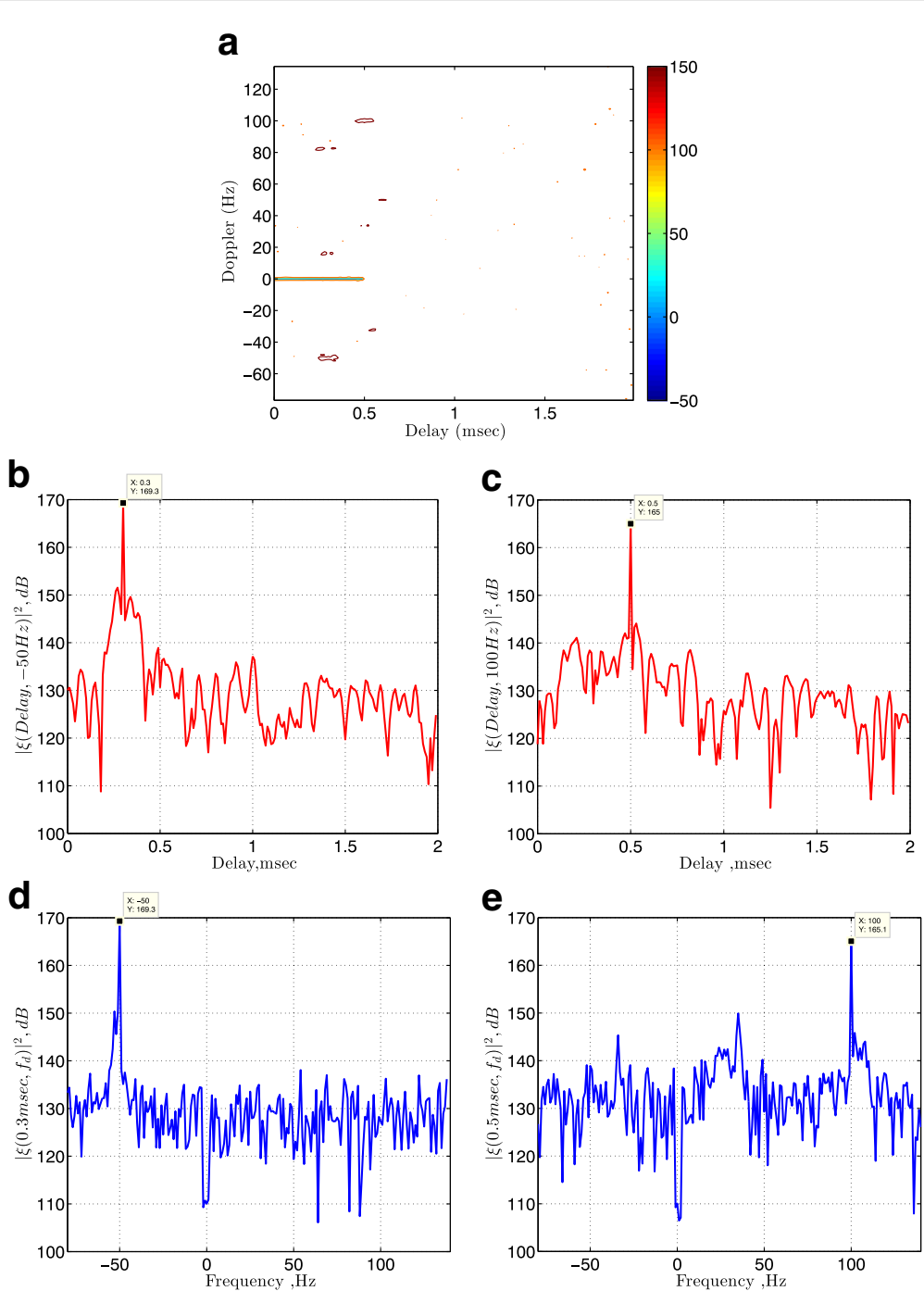

Fig. 8 The ambiguity function output in $\mathrm{dB}$ after direct signal and all clutter cancellation by the SCB algorithm with Doppler bin $(-1,0,1)$ in scenario \#1 a. 2-D output. b Section at Doppler $-50 \mathrm{~Hz}$. c Section at Doppler $100 \mathrm{~Hz}$. d Section at delay $0.3 \mathrm{~ms}$. e Section at delay $0.5 \mathrm{~ms}$

TA tests, we consider scenario \#2 containing one target and one clutter (spot clutter1 or exponential spectrum clutter2) with characteristics tabulated in Table 4.

According to scenario \#2, we obtain the values of CA and TA of the algorithms. For clutter and direct signal cancellation, $p$ is considered as $-1,0$ and 1 . Since the $\mathrm{CA}$ and TA of SCB algorithm depend on the number of batches $(b)$, we consider these quantities for various values of $b$. In Fig. 10, a simulated CA curve of the $\mathrm{SCB}$ algorithm is depicted versus the number of batches in comparison with that of the ECA, ECA-B and SCA algorithms. It is observed that the CA of SCB and ECA-B is similar, and when the clutter has an exponential spectrum, the CA is reduced by $6 \mathrm{~dB}$ (in both SCB and ECA-B methods). It is seen that the $\mathrm{CA}$ of $\mathrm{SCB}$ with ten batches ( $b=10)$ is close to that of ECA and SCA. By increasing $b$ from 10 to 30 , the CA of SCB increases. For $b$ more than 30, further attenuation for clutter is no longer available.

Figure 11 shows a TA curve of SCB algorithm versus the number of batches in comparison with that of ECA, SCA and ECA-B algorithms. It is seen that in the SCB algorithm with $b$ less than ten batches, similar to the ECA and SCA, the amplitude of the target in the ambiguity function almost is not reduced after clutter/direct signal cancellation. Nevertheless, as seen in Fig. 11, the amplitude of the target after cancellation will be reduced by increasing the number of batches from ten. This may cause the target not to be detected from the ambiguity function. It is observed 


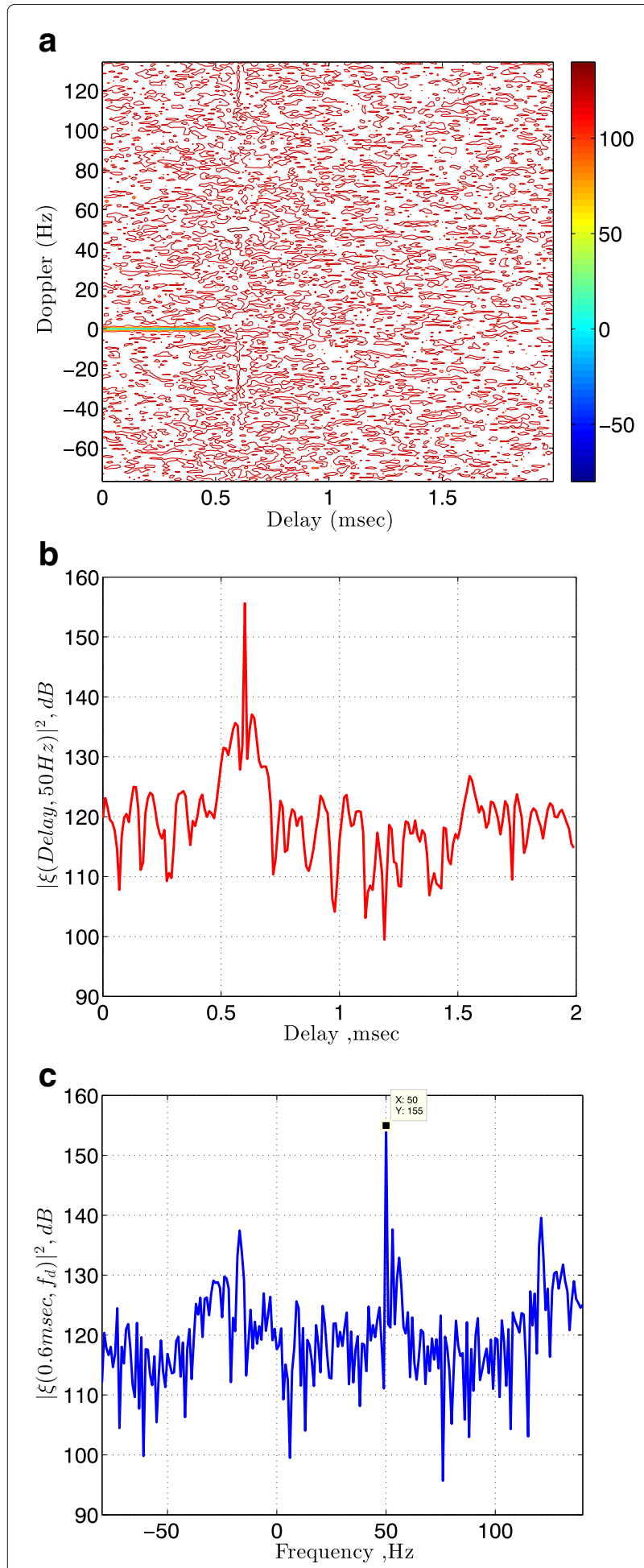

Fig. 9 The ambiguity function output in $\mathrm{dB}$ after direct signal, all clutters, and strong target cancellation by SCB algorithm in scenario \#1. a 2-D output. b Section at Doppler $50 \mathrm{~Hz}$. c Section at delay $0.6 \mathrm{~ms}$

that the TA of SCB and ECA-B is similar, and when the clutter has an exponential spectrum, the TA is not changed.
Table 4 Clutter and target parameters in scenario \#2 for calculation of CA and TA in SCB, ECA and SCA algorithms

\begin{tabular}{llll}
\hline & Delay $(\mathrm{ms})$ & Doppler $(\mathrm{Hz})$ & SNR $(\mathrm{dB})$ \\
\hline Clutter1 & 0.25 & 0 & 25 \\
Clutter2 & 0.25 & $\begin{array}{l}\text { Exponential Spectrum } \\
\text { between }-1 \text { and } 1 \mathrm{~Hz}\end{array}$ & 25 \\
& & 100 & 5 \\
\hline
\end{tabular}

\subsection{Evaluation using CFAR target detection}

In this section, in order to evaluate the proposed algorithm based on the target detection criteria, we use a CACFAR detector after clutter and direct signal cancellation using the mentioned algorithms. We use receiver operating characteristic (ROC) curves for detection performance comparison. In this manner, first, clutter and direct signal are removed by the SCB (or ECA and ECA-B) algorithm, and then targets are detected based on the output of ambiguity function and CA-CFAR detector. The detectors based on the ECA, ECA-B and SCB algorithms are called ECA-CA, ECA-B-CA and SCB-CA, respectively.

For comparing the ECA-CA, ECA-B-CA and SCB$\mathrm{CA}$ algorithms, we consider scenario \#3 where targets have been placed according to Table 5 in Delay-Doppler page and there are nine clutters with $f_{c}=0$ (or one clutter with exponential spectrum between -1 and $1 \mathrm{~Hz}), 5 \mathrm{~dB}<C N R<30 \mathrm{~dB}$ and maximum delay $0.3 \mathrm{~ms}$, where $\mathrm{CNR}$ denotes clutter-to-noise ratio.

In Fig. 12, the curves of detection probability $P_{d}$ versus SNR of ECA-CA, ECA-B-CA and SCB-CA detectors are plotted for nominal probability of false alarm $P_{f a}=0.01$.

It is observed that the ROC of SCB and ECA-B is similar, and when the clutter has an exponential spectrum SNR is reduced to $4 \mathrm{~dB}$ in both methods. It is

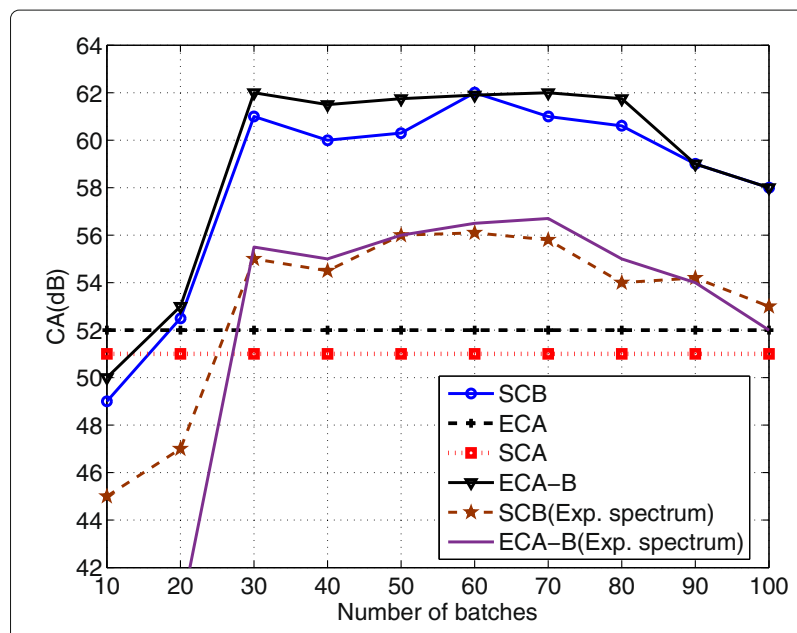

Fig. 10 The CA curve of SCB versus the number of batches in comparison with that of ECA, ECA-B and SCA in scenario \#2 


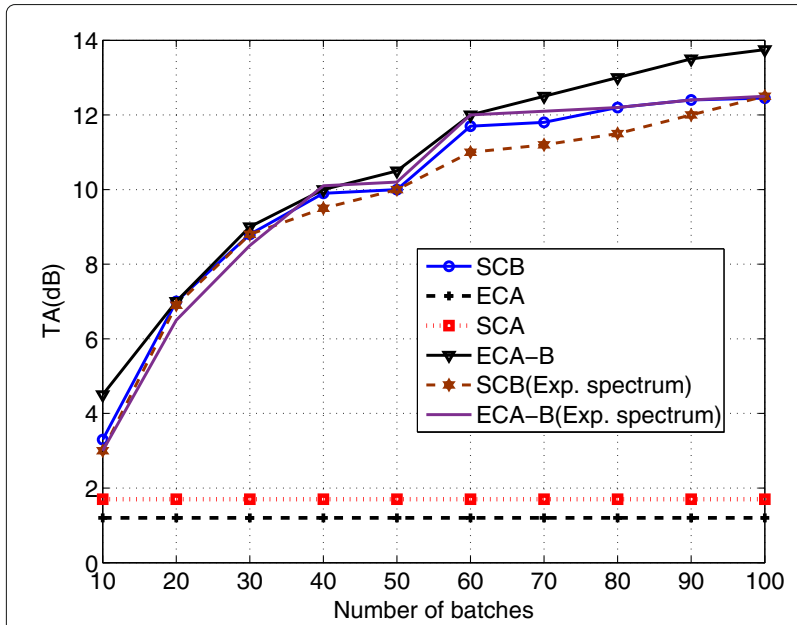

Fig. 11 The TA curve of SCB versus the number of batches in comparison with that of ECA, ECA-B and SCA in scenario \#2 seen that by decreasing the number of batch in the $\mathrm{SCB}$ algorithm, the performance of the SCB-CA improves so that the ROC of SCB-CA with $b=10$ is close to the ROC of ECA for both targets $T_{1}$ and $T_{2}$. This means the SCB-CA detector performs similar to the ECA-CA detector if the number of batch is low. Consider that the SCB-CA has less computational complexity than that of ECA-CA. The ECA-CA and SCB-CA detectors degrade if Doppler frequency of target tends to be $0 \mathrm{~Hz}$.

\section{Conclusions}

In this paper, the SCB algorithm is proposed for cancellation of static and non-static clutters as well as elimination of direct signal component in passive bistatic radars based on projections of the received signals onto a subspace orthogonal to the signal subspace of the clutter and the subspace of the previously detected targets. The SCB algorithm is first used for clutter and direct signal cancellation and detection of strong targets. To enhance the detection performance, the observation algorithm is then investigated and applied for detection of targets with weak signals. The simulation results revealed that the SCB algorithm performers well in the detection of targets compared with the state-of-the-art methods. The TA, CA and CFAR detection tests were used for comparing the SCB with the ECA, ECA-B and SCA algorithms. These tests

Table 5 Clutter and target parameters in scenario \#2 for calculation of CA and TA in SCB, ECA, ECA-B and SCA algorithms

\begin{tabular}{lll}
\hline & Target $T_{1}$ & Target $T_{2}$ \\
\hline Delay (ms) & 0.1 & 0.9 \\
Doppler $(\mathrm{Hz})$ & 10 & 50 \\
\hline
\end{tabular}

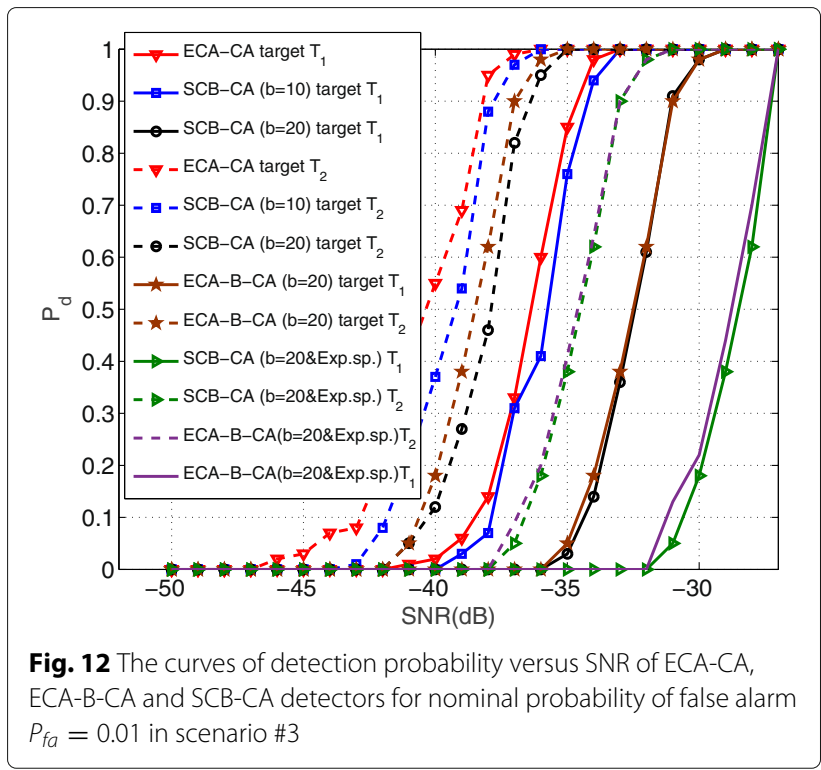

showed that targets may hide in the ambiguity function when the number of batches increases. The SCB algorithm has lesser computational complexity than the ECA and ECA-B algorithms. Moreover, the proposed method requires lesser memory than these algorithms and the SCA method.

\section{Competing interests}

The authors declare that they have no competing interests.

\section{Author details}

${ }^{1}$ Department of Electrical and Computer Engineering, Yazd University, 89195-741 Yazd, Iran. ${ }^{2}$ Department of Electrical and Computer Engineering, Isfahan University of Technology, Isfahan 84156-83111, Iran. ${ }^{3}$ Department of Electrical and Computer Engineering, Queen's University, 99 Union St., Kingston ON K7L 3N6, Canada.

Received: 24 July 2016 Accepted: 25 November 2016

Published online: 09 December 2016

\section{References}

1. HD Griffiths, NR Long, Television based bistatic radar. IEE Proc. Part F Commun. Radar Signal Process. 133, 649-657 (1986)

2. F Ansari, MR Taban, Clutter and direct signal cancellation in analog TV-based passive radar. Radar. 1, 1-14 (2014)

3. A Lauri, F Colone, R Cardinali, P Lombardo, in Proceedings of IEEE International Conference on Aerospace. Analysis and emulation of FM radio signals for passive radar (IEEE, Big Sky, MT, 2007)

4. M Cherniakov, in Proceedings of IET International Conference on Radar. Space-surface bistatic synthetic aperture radar prospective and problems (IET, Edinburgh, 2002)

5. HA Harms, LM Davis, J Palmer, Understanding the signal structure in DVB-T signals for passive radar detection. (IEEE, Washington, 2010)

6. D Poullin, Passive detection using broadcasters (DAB, DVB) with CODFM modulation. IEE Proc. Radar Sonar Navigation. 152, 143-152 (2005)

7. D Tan, H Sun, Y Lui, M Lesturgie, H Chan, Passive radar using global system for mobile communication signal: theory, implementation and measurements. IEE Proc. Radar Sonar Navigation. 152, 116-123 (2005)

8. PE Howland, D Maksimiuk, G Reitsma, FM radio basedbistatic radar. IEE Proc.Radar Sonar Navigation. 152, 107-115 (2005)

9. T Tsao, M Slamani, P Varshney, D Weiner, H Schwarzlande, Ambiguity function for a bistatic radar. IEEE Trans. Aerospace Electronic Syst. 33, 1041-1051 (2009) 
10. A Zaimbashi, M Derakhtian, A Sheikhi, GLRT-based CFAR detection in passive bistatic radar. IEEE Trans. Aerospace Electron. Syst. 49, 134-159 (2013)

11. A Zaimbashi, M Derakhtian, A Sheikhi, Invariant target detection in multiband FM-based passive Bistatic radar. IEEE Trans. Aerospace Electron. Syst. 50, 720-736 (2014)

12. J Palmer, SJ Searle, in Proceedings of IEEE International Conference on Radar. Evaluation of adaptive filter algorithms for clutter cancellation in passive bistatic radar (IEEE, Atlanta, 2012)

13. F Wang, in Proceedings of IET International Conference on Radar. Direct signal recovery and masking effect removal exploiting sparsity for passive bistatic radar (IET, Hangzhou, 2015)

14. F Colone, R Cardinali, P Lombardo, in Proceedings of IEEE International Conference on Radar. Cancellation of clutter and multipath in passive radar using a sequential approach (IEEE, Verona, 2006)

15. F Colone, DW O'Hahan, P Lombardo, CJ Baker, A multistage processing algorithm for disturbance removal and target detection in passive bistatic radar. IEEE Trans. Aerospace Electron. Syst. 45, 698-722 (2009)

16. A Ansari, MR Taban, in Proceedings of Iranian Conference on Electrical and Electronic Engineering (ICEE2013). Implementation of sequential algorithm in batch processing for clutter and direct signal cancellation in passive bistatic radars (IEEE, Mashhad, 2013)

17. F Colone, C Palmarini, T Martelli, E Tilli, Sliding extensive cancellation algorithm (ECA-S) for disturbance removal in passive radar. IEEE Trans. Aerospace Electron. Syst. 52, 1326-1309 (2016)

18. K Kulpa, in Proceedings of Microwaves, Radar and Remote Sensing. The clean type algorithms for radar signal processing (IEEE, Kiev Ukraine, 2008)

19. RD Fry, DA Gray, in Proceedings of International Conference on Radar. Clean deconvolution for sidelobe suppression in random noise radar (IEEE, Adelaide, 2008)

20. C Jinli, Z Xiuzai, L Jiaqiang, G Hong, in Proceedings of IEEE International Conference on Information Science and Technology (ICIST). Ground clutter suppression using noise radar with dual-frequency transmitter (IEEE, Hubei, 2012)

21. K Kulpa, Z Czekala, Masking effect and its removal in PCL radar. I EE Proc. Radar Sonar Navigation. 152, 174-178 (2005)

\section{Submit your manuscript to a SpringerOpen ${ }^{\circ}$ journal and benefit from:}

- Convenient online submission

- Rigorous peer review

- Immediate publication on acceptance

- Open access: articles freely available online

- High visibility within the field

- Retaining the copyright to your article

Submit your next manuscript at $\gg$ springeropen.com 